\title{
Differential expression of Ago2-mediated microRNA signaling in adipose tissue is associated with food induced obesity
}

Xin Yan ( $\nabla$ xin.yan@xxmu.edu.cn )

Xinxiang Medical University

Hansi Zhang

Xinxiang Medical University

Liang Qiao

Xinxiang Medical University

Xiaoxuan Liu

Xinxiang Medical University

Xiaojing Han

Xinxiang Medical University

Jing Kang

Xinxiang Medical University

Yanli Liu

Xinxiang Medical University

Juntang Lin

Xinxiang Medical University

\section{Research Article}

Keywords:

Posted Date: March 8th, 2022

DOI: https://doi.org/10.21203/rs.3.rs-1355723/v2

License: (c) (1) This work is licensed under a Creative Commons Attribution 4.0 International License. Read Full License 


\section{Abstract}

Adipose tissue is a major component for the regulation of energy homeostasis by storage and release of lipids. As a core element of RNA-induced silencing complex, Ago2 plays critical role in maintenance of systemic metabolic demand. Here we show that high-fat diet feeding mice exhibited an increase in body mass alongside systematic insulin resistance and altered rate of energy expenditure. Interestingly, Ago2 expression is associated with obesity and increased in adipose tissues. Moreover, increase of Ago2 inhibited the expression of AMPKa by promoting its targeting by miR-148a, the most abundant microRNA in the adipose tissues. Those results suggested that Ago2-miR-148a-AMPKa signaling pathway play an important function in the developing obesity and adiposity and will further provide basic research data for the potential clinic treatment of obesity.

\section{Introduction}

Obesity is a chronic progressive disease and characterized by energy imbalance resulting from excessive calorie intake and abnormal energy expenditure ${ }^{1}$. Generally, it's associated with type 2 diabetes, insulin resistance and inflammation ${ }^{2,3}$. As the prevalence of the disease continues to increase worldwide, it remains imperative to identify novel genes and their pathways that contribute to obesity. Recent studies have shown that several tissue specific microRNAs (miRNA) contributed to the progression of obesity, dissecting their mechanism of action in vivo still remain a challenge.

The Argonaute 2 (Ago2) is a core component of RNA-induced silencing complex (RISC) and can mediate miRNA to interact with target $m R N A A^{4,5}$. Interestingly, Ago2 related miRNA pathway is emerging as an important contributor in several tissues and essential to regulate glucose metabolism and cellular energy homeostasis $6,7,8,9$. For example, Ago2 is considered to be an important regulator of liver energy metabolism and high-fat diet feeding forces liver to express excessive Ago2 in liver ${ }^{8}$. Conditional deletion of Ago2 in liver can improve the glucose metabolism through the activation of AMPK signaling pathway during high-fat diet (HFD) feeding ${ }^{8,9}$. Furthermore, obesity significantly increases the expression of Ago2 in islets, giving rise to pancreatic $\beta$-cell proliferation and insulin resistance, which further provide evidence for Ago2 in the regulation of cellular metabolism ${ }^{6}$.

Adipose tissues have an important function in sensing and managing energy status through lipid storage and release, and adipokines secretion ${ }^{10,11}$. Strongly accumulation of excess visceral adiposity can cause obesity which is associated with different metabolic dysfunction including chronic inflammation ${ }^{11,12}$. Several studies have reported that many miRNAs in adipose tissues including miR-34a, miR-93, miR-106, miR-148a, miR-221 have been shown to affect adipogenesis and adiposity ${ }^{13,14,15,16,17}$. For example, miR93 has been shown to control adipocyte differentiation by negatively regulating $T b \times 3$, loss of miR-93 results in increased fat mass and insulin resistance ${ }^{14}$. Furthermore, miR-148a is required for the adipocyte differentiation and highly increased in the epididymal adipose tissue of mice after HFD 
feeding ${ }^{15}$. These results provide strong evidence for the miRNA pathway in regulating cellular energy metabolism in the adipose tissues.

Several studies have shown that tissue specific Ago2 and its mediated miRNA signaling pathways play a crucial role on body metabolism and contribute to the progression of obesity. However, it is unclear whether this function extends to other tissues, especially in adipose tissue. Therefore, current study will investigate the role of Ago2 in fat tissues. We find that HFD-induced obesity is defined by the metabolic dysregulation and disrupted energy homeostasis, and that these changes are largely associated with the evaluated expression of Ago2 in different adipose depots. The increased Ago2 led to a concomitant upregulation of miR-148a and downregulation in AMPKD expression in adipose tissues. Finally, histological analysis and thermogenesis genes were performed to assess their relationships to HFDinduced changes in miRNA signaling pathway. Together, those results demonstrate Ago2 as an important regulator in physiological processes of adipose tissues and further reinforce the role of Ago2-mediated miRNA signaling in regulation of HFD-induced obesity.

\section{Materials And Methods}

\section{Animals model}

All animals maintained on a $12 \mathrm{~h}$ light/dark cycle. To obtain high fat diet induced obesity model, C57/BL6J mice (6 weeks old) were randomly assigned to two groups. Then, the mice were fed either normal chow diet (Chow) with $10 \mathrm{KJ} \%$ fat (Keao Xieli, Beijing) as control group, or high fat diet (HFD) with $60 \mathrm{KJ} \%$ fat (Keao Xieli, Beijing) as obesity group ad libitum until 16 weeks. Before adipose tissue was collected, the mice were anesthetized with isoflurane and sacrificed after cervical dislocation.

All animal experiments were carried out in accordance with Chinese Council on Animal Care guidelines, and the experimental protocol was approved by the Animal Research Committee of Xinxiang Medical University. Additionally, all methods were performed in accordance with the relevant guidelines and regulations and the study was carried out in compliance with the ARRIVE guidelines.

\section{Body weight and composition analysis}

Body weights were measured every week with a digital scale. Body composition of lean body mass (LBM) and fat mass were measured using time-domain nuclear magnetic resonance (TD-NMR) method (the minispec Live Mice Analyzer LF90, Bruker).

\section{Analysis of glucose metabolism in mice}

Blood samples were collected from submandibular vein and centrifuged at $2000 \mathrm{~g}$ for $10 \mathrm{~min}$. Plasma insulin and leptin were measured by commercial enzyme-linked immunosorbent assay (ELISA) kit 
(Crystal Chem Inc. \#90080, \#90030). Plasma triglyceride, free fat acid (FFA), glycerol, and cholesterol levels were measured using commercially available kits (Cayman, 10010303, 700310, 10010755, 10007640).

For glucose tolerance test (GTT), mice were injected intraperitoneally (i. p.) with D-glucose $(2 \mathrm{mg} / \mathrm{g}$ body weight) after overnight fasting, blood glucose levels were measured using Glucometer (Contour, Bayer) before and following the injection from the facial vein of non-anesthetized animals. For insulin tolerance test (ITT), $6 \mathrm{~h}$ fasted mice were injected by i. p. with human recombinant insulin $(0.75 \mathrm{U} / \mathrm{kg}$ body weight, PAN Biotech), and tail-tip blood glucose was measured before and after the injection.

\section{Metabolic Phenotyping analysis}

Sixteen weeks old Chow and HFD mice were acclimated into home cage of Promethion Core System (Sable Systems International) for $24 \mathrm{~h}$ before the start of the experimental record. Then, $\mathrm{O}_{2}$ and $\mathrm{CO}_{2}$ exchange were continuously recorded every $9 \min$ for 1 min for 4 days, and locomotor activity was continuously recorded by interruptions of infrared light beams. $\mathrm{O}_{2}$ consumed $\left(\mathrm{VO}_{2}\right), \mathrm{CO}_{2}$ produced $\left(\mathrm{VCO}_{2}\right)$, and energy expenditure (EE) were calculated following the manufacture's manual, and the respiratory exchange ratio (RER) was computed by the ratio of $\mathrm{VCO}_{2} / \mathrm{VO}_{2}$. Finally, EE was analyzed either after the normalization by LBM or locomotor activity using covariance (ANCOVA).

\section{Western blot}

Total protein of brown adipose tissue (BAT), subcutaneous white adipose tissues (sWAT), and epididymal white adipose tissues (eWAT) were lysed by RIPA buffer, and quantitated by Pierce ${ }^{\mathrm{TM}}$ BCA Protein Assay kit $(23225$, ThermoFisher Scientific). Equal amounts of protein $(20 \mu \mathrm{g})$ were separated by SDS-PAGE electrophoresis, transferred to PVDF membrane, and blocked in 5\% skim milk in TBST. Then, the membrane was incubated overnight with primary antibody solution at $4{ }^{\circ} \mathrm{C}$. After rinsing the blot, the membrane was incubated in the horseradish peroxidase (HRP)-conjugated secondary antibody solution for $2 \mathrm{~h}$ at room temperature. Finally, chemiluminescent signals on the blot were applied with SuperSignal ${ }^{\mathrm{TM}}$ West Femto Maximum Sensitivity Substrate (34095, ThermoFisher Scientific), and captured using Amersham Imager 600 imagers (GE Healthcare Life Sciences). The band intensity of protein was read by image analysis software Fiji. The following primary and secondary antibodies were used in this study: Ago2 (RN003M, MBL International, 1:1000), UCP1 (\#14670, Cell Signaling Technology, 1:1000), AMPKa (\#2532, Cell Signaling Technology, 1:1000), p-AMPKa (\#2535, Cell Signaling Technology, 1:1000), GAPDH (ab8245, Abcam, 1:1000), Goat Anti-Rabbit IgG H\&L (HRP) (ab6721, Abcam, 1:10000), Goat Anti-Mouse IgG H\&L (HRP) (ab6789, Abcam, 1:10000).

\section{Quantitative real-time polymerase chain reaction (qPCR)}


Total RNA was isolated from BAT, sWAT, eWAT and inguinal white adipose tissue (ingWAT) using RNeasy plus Mini kit (QIAGEN) and quantitated by Spark ${ }^{\circledR}$ (TECAN). First strand CDNA from total RNA was efficiently synthesized using RevertAid First Strand cDNA Synthesis Kit (\#K1621, ThermoFisher

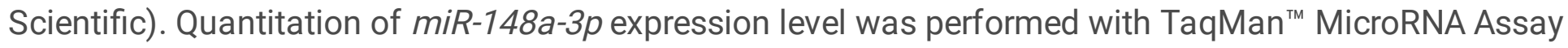
(4440887, Applied Biosystems) and normalized to U6 snRNA following the primers from Applied Biosystems: mmu-miR-148a-3p (mmu477814_mir) and U6 snRNA (001973). qPCR of mRNAs was detected using FastStart SYBR Green Master (04673484001, Roche) and normalized to $\beta$-actin in StepOne $^{\text {TM }}$ Real-Time PCR Systems (Applied Biosystems). The sets of primer sequence were ordered from ThermoFisher Scientific in Supplementary Table S1.

\section{Cell culture}

The HEK293T cell line was obtained from CCTCC (1101HUM-PUMC000091, Beijing). Cells were cultured in Dulbecco's modified Eagle's medium (DMEM; 10569010, Gibco) supplemented with $10 \%$ fetal bovine serum (FBS; abs972, Absin) and 1\% penicillin/streptomycin (15140122, Gibco) in a 5\% CO2 atmosphere at $37^{\circ} \mathrm{C}$.

\section{Luciferase assay and transfection}

The 3'-UTR of murine AMPKa was PCR amplified using the following primers 5'-TGG TAG CAT AGC ATA ATG GG-3' and 5' -CAA CAG TTT ATA GAG ATA TTC CTC AG-3' and cloned into the pGL3 Luciferase Reporter Vectors (Promega). HEK293T cells were seeded for co-transfection of AMPKa 3'-UTR plasmid and miR-148a mimics (MC10263, ThermoFisher Scientific) or mirVana ${ }^{\text {TM }}$ miRNA Mimic, Negative Control \#1 (4464058, ThermoFisher Scientific). After $48 \mathrm{~h}$ of transfections, luciferase assays were detected using the Dual-Luciferase ${ }^{\circledR}$ Reporter Assay System (Promega) following the manufacturer's manual.

\section{Histological detection}

The adipose tissue pads and liver were dissected from 16-week-old mice, fixed in $4 \%$ paraformaldehyde (PFA), and embedded in paraffin. Tissue sections were stained with hematoxylin and eosin (H\&E) and imaged using a Nikon ECLIPSE $80 \mathrm{i}$ microscope.

\section{Statistical analyses}

All experimental data were expressed as mean \pm standard error (SEM), statistical analysis was performed using student's $t$ test, two-way ANOVA in GraphPad Prism 8, and ANCOVA in SPSS. $P<0.05$ was considered statistically significant (see Supplementary Table S2 online.). 


\section{Results}

\section{High fat diet induces obesity}

To observe HFD-induced obesity, C57/BL6J mice were divided into two groups and fed with normal chow diet or high fat diet from 6-week-old. Body weight was monitored weekly until 16-week-old age. Interestingly, administration of the high fat diet can significantly increase in body weight compared to chow diet controls starting at 8-week-old age (Fig. 1a). The increased body weight was likely due to an increase in fat mass as shown by NMR analysis of body composition at 16 weeks of age (Fig. 1b, c). Consistent with increased adiposity, plasma levels of leptin were strongly increased almost fifteen-fold in HFD mice compared to normal chow diet fed mice (Fig. 1d). Due to the association of obesity with diabetes and insulin resistance, we further analyzed the systemic glucose metabolism in HFD fed mice and normal chow diet fed mice. Both random and fasting plasma glucose and insulin levels were elevated in HFD mice (Fig. 1e, f). Glucose and insulin tolerance tests (GTT and ITT) showed higher glucose levels in HFD mice in comparison to chow diet fed controls indicating systemic insulin sensitivity was reduced (Figure. 1g, h). Lastly, the determination of plasma levels of various hormones including triglyceride, FFA, glycerol and cholesterol were also significantly elevated in the HFD mice (Fig. i-I).

\section{Changes in basic metabolic rates in HFD feeding mice}

To understand the factors which may contribute to the increase in body mass and adiposity, we next determined energy metabolism in these animals in HFD animals. Indirect gas calorimetry was used to calculate basic metabolic rates in these animals at 12-week-old age. As shown in Fig. 2, HFD mice exhibited no changes in oxygen consumption (Fig. 2a), but decreases in carbon dioxide production (Fig. 2b). Energy expenditure was slightly increased in HFD mice (Fig. 2c). These results translate into a decrease in the nutrient partitioning (based on respiratory exchange rate (RER) measured in HFD mice and suggest fat to be the predominant energy source (Fig. 2d). Additionally, the spontaneous locomotor activity was reduced in HFD mice (Fig. 2e). Moreover, energy expenditure, when plotted in relation to lean body mass in an ANCOVA, is again increased in HFD mice (Fig. 2f). However, this difference disappears when energy expenditure is plotted in relation to locomotor activity (Fig. $2 \mathrm{~g}$ ). These results suggested that HFD animals of similar lean body mass to controls have increased energy expenditure is directly correlated to the shift in metabolic fuel from carbohydrates in normal chow diet to lipids in high fat diet.

\section{High fat diet increases Ago2 and miR-148a expression in the adipose tissue}

Recent studies have revealed that Ago2-miRNA-mediated mRNA regulation plays a significant role in the hepatic and adipose energy metabolism of obesity ${ }^{9}$. Due to miRNA dysregulation in the large repertoire of physiological processes of obesity ${ }^{18}$, we sought to examine the expression of Ago2-mediated signaling in 
obese adipose tissue. Western blot analysis indicated an increased Ago2 protein levels in adipose tissue of HFD mice compared to normal chow diet fed control (Fig. 3a). Consistent with increased body fat mass, Ucp 1 expression is reduced in BAT and subcutaneous sWAT of HFD mice (Fig. 3a). Meanwhile, qPCR analysis further indicated increased Ago2 mRNA in different fat tissues after HFD feeding (Fig. 3b). As miR-148a has been involved in the energy metabolism ${ }^{8}$, the expression level of miR-148a was checked by qPCR. Consistent with the Ago2, miR-148a expression levels were significantly increased in BAT, sWAT, and eWAT of HFD mice compared to normal chow diet fed control mice (Fig. 3c), these results support the critical role of Ago2 in the expression of miR-148a.

\section{Ago2 regulates miRNA-mediated targeting of $A M P K a$}

To elucidate the molecular basis for the role of Ago2 in adipose tissues, we next sought to identify candidate target genes that are involved in the lipid metabolism. We first hypothesized that AMPKa may be subject to Ago2: miRNA-mediated gene regulation in the adipose tissue in light of its prominent role in lipid metabolism as well as its establish role as an energy and metabolic sensor in response to changes in nutrient and hormone availability ${ }^{19,20}$. Importantly, AMPKd's 3' UTR has been reported to have an miR148/152 target site ${ }^{8}$. To establish the direct interaction between Ago2, miR-148a, and the AMPKa mRNA, we performed luciferase assays in which $A M P K d$ 's 3' UTR was cloned into luciferase expression vector. The luciferase activity of each was measured in HEK293 cells. Comparing to control miRNA mimics, miR148a mimics can strongly inhibit the luciferase activity (Fig. 4a). Western blots also indicated a decreased protein level of AMPKa after transfection of miR-148a mimics (Fig. 4b). We next measured expression of $A M P K a$ in different adipose tissues and identified the down-regulation of AMPKa mRNA after HFD feeding (Fig. 4c). Western blot confirmed a similar decrease in both total AMPKa protein and pAMPKa in HFD mice compared to normal chow diet control mice (Fig. 4d), which is associated with a decreased AMPK activity represented as the ratio of p-AMPKa/AMPKa in the fat tissues of HFD mice (Fig. 4e). Together, those data indicated that miR-148a is involved in suppression of AMPKa expression in a manner dependent on Ago2 and a miR-148a target site.

\section{High fat diet causes the lipid accumulation by changing lipid metabolism}

To further determine the causes for the increased body weight, we next performed histological analysis in the BAT, ingWAT, eWAT and liver. As expected, hematoxylin and eosin stain revealed a massive lipid accumulation in HFD fed BAT compared to normal chow diet mice (Fig. 5a). Consistent with this observation, gene expression analysis showed HFD increased Elov/3 expression, which is involved in fat cell elongation, but decreased expression of genes involved in BAT thermogenesis and oxidation (including Ucp1, Cidea, Cox8b) (Fig. 5b). Histological analysis of WAT tissue suggests a lipid hypertrophy in fat cells of HFD mice (Fig. 5c, e), and gene expression analysis showed significantly increased expression of Elov/3 of HFD mice (Fig. 5d, f). Furthermore, expression analysis in ingWAT of BAT enriched 
genes was lower notablely in HFD mice compared with chow mice (Fig. 5d), but in eWAT was unaltered (Fig. 5f). These data suggest that HFD leaded to the loss of beige fat cells in ingWAT, which almost exists exclusively in subcutaneous depots. Finally, image analysis of liver also shows strongly increased lipid accumulation in liver (Fig. 5g). Consistently, the gene expression of Cidea increased significantly in liver (Fig. 5h). Also, the expression of $\beta$-oxidation-related genes in mitochondria such as UCP1, UCP2 and Cpt1 decreased (Fig. 5h, i). The increased expression of Elov/3, Cpt1 and Cd36 suggested increased synthesis of fatty acids and triglyceride, and free fatty acid uptake, respectively (Fig. 5h, i). The expression of genes related to de novo fatty acids synthesis such as Fas, and ACC1 showed decreased expression (Fig. 5i), which implicated the hepatic lipid accumulation induced by HFD was not caused by the increase of de novo fatty acids synthesis, but more likely due to the ectopic lipid deposition triggered by the excessive fat storage in adipose tissue.

\section{Discussion}

Numerous studies have described mechanisms which illustrate the contribution of the miRNA signaling in maintaining the balance between energy intake and expenditure. In the current study, we show that mice feeding with HFD exhibit diet-induced obesity and decreases in glucose homeostasis, insulin sensitivity and energy expenditure rate. Mechanistically, our measurements further show HFD induced obesity resulted in an alteration in expressions of Ago2-mediated miRNA signaling in adipose tissues. This causes suppression of miR-148a target gene $A M P K a$, which is an important nutrient and energy sensor in maintaining energy homeostasis. Those results are in line with previous studies showing Ago2 and miRNAs are involved adipogenesis ${ }^{15,21}$ and emphasize an important role for Ago2 in regulation of body homeostasis and fat adiposity.

Ago2, as a component of the miRNA-induced silencing complex, has been recently shown to be involved in the metabolic regulation because of its effects on insulin resistant and glucose metabolism ${ }^{6,8}$. Ago2 shows benefical impacts on obesity and insulin resistance by promoting pancreatic $\beta$ cell expansion ${ }^{6}$. Notably, liver Ago2 levels are elevated in the obese mice ${ }^{7}$, hepatic Ago2-deficiency improves glucose metabolism in conditions of high-fat diet challenge ${ }^{8}$, suggesting that Ago2 signaling might be impaired in different organs during obesity. Our data indicate that Ago2 expression in adipose tissues is increased in diet-induced model of obesity, in consistent with the role of Ago2 in obesity.

Furthermore, recent studies showed that several miRNAs controlled by Ago2 are key regulator of adiposity and involved in biological functions of adipogenesis. For example, miR-148a is a key miRNA and its expression gradually increased in the process of controlling human adipose-derived mesenchymal stem cells differentiation into adipocytes through binding to the target gene WNT1 ${ }^{15,21}$. Loss of miR-93 can increase adipose cell differentiation, fat mass and subsequently insulin resistance by targeting Tbx 3 gene ${ }^{14}$. Furthermore, miR-34a has been found to be increased which is associated with obesity and inhibit fat cell browning; Downregulation of miR-34a can elevate expression of FGF21 and results in 
induction of the browning genes $U c p 1, P g c-1$, and $P r d m 16^{22}$. Consistent with above studies is our observation showing an increase in expression of miR-148a in the adipose tissues of HFD fed mice.

A common characteristic of obesity is high circulating lipid levels, partly accounted by impaired insulinmediated suppression of lipolysis. AMPKa, an important cellular energy sensor, has been implicated in control of whole-body adiposity through regulation of lipolysis ${ }^{23}$. For example, AMPK inhibits lipogenesis in isolated adipocytes via increased ACC phosphorylation in response to AICAR stimulation ${ }^{24}$, while enhanced activation of AMPK can promote lipolysis ${ }^{25}$. While knockout of AMPKa in mice caused an increased body weight and fat mass due to the enlarged adipocytes and lipid accumulation in fat cells ${ }^{26}$. Consequently, we speculated that the regulation of adipose tissue by Ago2 is realized through Ago2-miR148a-AMPKa signaling pathway. Obesity induces the increase of Ago2 expression, which enhances the silencing effect of miR-148a on $A M P K a$, and subsequently reduces the activity of $A M P K a$, inhibits the calorie burning ability of adipocytes, and finally leads to lipid accumulation.

\section{Conclusion}

In conclusion, our observations on the Ago2 provide further insight into identifying a specific miRNA signaling which regulate obesity and energy homeostasis in adipose tissue. Improving our understanding of Ago2 in adiposity and lipid metabolism may facilitate mapping of distinct systemic networks to delineate the unique properties of separate cell populations contributing to metabolism. It will be important to further investigate mechanism of how Ago2 and related miRNAs in the progression of dietinduced obesity.

\section{Abbreviations}

ACC1: Acetyl-Coenzyme A carboxylase alpha; ACC2: Acetyl-Coenzyme A carboxylase beta; Ago2: Argonaute 2; BAT: Brown adipose tissue; AMPKa1: Protein kinase, AMP-activated, alpha 1 catalytic subunit; Cd36: CD36 molecule; Chow: Chow diet; Cidea: Cell death-inducing DNA fragmentation factor, alpha subunit-like effector $A$; ChREBP: MLX interacting protein-like; Cox8b: Cytochrome c oxidase subunit 8B; Cpt1: Carnitine palmitoyltransferase 1a; EE: Energy expenditure; ELISA: Enzyme-linked immunosorbent assay; Elovl3: Elongation of very long chain fatty acids; eWAT: Epididymal white adipose tissue; Fas: Fatty acid synthase; FFA: Free fat acid; GPAT: Glycerol-3-phosphate acyltransferase, mitochondrial; GTT: Glucose tolerance test; HFD: High-fat diet; HMGCR: 3-hydroxy-3-methylglutarylCoenzyme A reductase; ingWAT: Inguinal white adipose tissue; ITT: Insulin tolerance test; LBM: Lean body mass; miRNA: microRNA; RER: Respiratory exchange ratio; Ldlr: Low density lipoprotein receptor; RISC: RNA-induced silencing complex; S14: SPOT14, Thyroid hormone responsive; Srebp1: Sterol regulatory element binding transcription factor 1 ; sWAT: Subcutaneous white adipose tissue; TD-NMR: Time-domain nuclear magnetic resonance; Ucp1: Uncoupling protein 1; Ucp2: Uncoupling protein 2

\section{Declarations}




\section{Funding}

This work was funded by the National Natural Science Foundation of China $(82071326,81870587)$, China Scholarship Council (201708410345), Foundation for the University Key Teacher by the Henan Province (2020GGJS148), Key Technologies R\&D Program of Henan Province (222102310086), Natural Science Foundation of Henan Province for Distinguished Young Scholars (202300410307), Graduate Research and Innovation Program of Xinxiang Medical University (YJSCX202001Z), College Students' Innovation and Entrepreneurship Training Program (202110472013).

\section{Data availability}

All data generated or analysed during this study are included in this published article (and its Supplementary Information files) and are available from the corresponding author on reasonable request.

\section{Author's contributions}

H.Z., L.Q., X.L., X.H., J.K., Y.L., J.L. and X.Y. contributed to the conception and design of the study, L.Q. and $X . Y$. wrote the manuscript. All authors approved the final version of this manuscript.

\section{Competing interests}

The authors declare no competing interests.

\section{References}

1. Blüher, M. Obesity: global epidemiology and pathogenesis. Nat. Rev. Endocrinol. 15, 288-298 (2019).

2. Piché, M.-E., Tchernof, A. \& Després, J.-P. Obesity Phenotypes, Diabetes, and Cardiovascular Diseases. Circ. Res. 126, 1477-1500 (2020).

3. Zhang, A. M. Y., Wellberg, E. A., Kopp, J. L. \& Johnson, J. D. Hyperinsulinemia in Obesity, Inflammation, and Cancer. Diabetes Metab. J. 45, 285-311 (2021).

4. Meister, G. Argonaute proteins: functional insights and emerging roles. Nat. Rev. Genet. 14, 447-459 (2013).

5. Müller, M., Fazi, F. \& Ciaudo, C. Argonaute Proteins: From Structure to Function in Development and Pathological Cell Fate Determination. Front. Cell Dev. Biol. 7, 360 (2019).

6. Tattikota, S. G. et al. Argonaute 2 mediates compensatory expansion of the pancreatic $\beta$ cell. Cell Metab. 19, 122-134 (2014).

7. Yan, X. et al. Control of hepatic gluconeogenesis by Argonaute2. Mol. Metab. 18, 15-24 (2018). 
8. Zhang, C. et al. Hepatic Ago2-mediated RNA silencing controls energy metabolism linked to AMPK activation and obesity-associated pathophysiology. Nat. Commun. 9, 3658 (2018).

9. Bhattacharjee, J. et al. Hepatic Ago2 Regulates PPARa for Oxidative Metabolism Linked to Glycemic Control in Obesity and Post Bariatric Surgery. Endocrinology 162, bqab007 (2021).

10. Peirce, V., Carobbio, S. \& Vidal-Puig, A. The different shades of fat. Nature 510, 76-83 (2014).

11. Reilly, S. M. \& Saltiel, A. R. Adapting to obesity with adipose tissue inflammation. Nat. Rev. Endocrinol. 13, 633-643 (2017).

12. Kawai, T., Autieri, M. V. \& Scalia, R. Adipose tissue inflammation and metabolic dysfunction in obesity. Am. J. Physiol. Cell Physiol. 320, C375-C391 (2021).

13. Wu, Y. et al. Identification of miR-106b-93 as a negative regulator of brown adipocyte differentiation. Biochem. Biophys. Res. Commun. 438, 575-580 (2013).

14. Cioffi, M. et al. MiR-93 Controls Adiposity via Inhibition of Sirt7 and Tbx3. Cell Rep. 12, 1594-1605 (2015).

15. Shi, C. et al. miR-148a is Associated with Obesity and Modulates Adipocyte Differentiation of Mesenchymal Stem Cells through Wnt Signaling. Sci. Rep. 5, 9930 (2015).

16. Peng, J. et al. miR-221 negatively regulates inflammation and insulin sensitivity in white adipose tissue by repression of sirtuin-1 (SIRT1). J. Cell. Biochem. 119, 6418-6428 (2018).

17. Pan, Y. et al. Adipocyte-secreted exosomal microRNA-34a inhibits M2 macrophage polarization to promote obesity-induced adipose inflammation. J. Clin. Invest. 129, 834-849 (2019).

18. Zaiou, M., El Amri, H. \& Bakillah, A. The clinical potential of adipogenesis and obesity-related microRNAs. Nutr. Metab. Cardiovasc. Dis. NMCD 28, 91-111 (2018).

19. Yan, C. et al. A High-Fat Diet Attenuates AMPK a1 in Adipocytes to Induce Exosome Shedding and Nonalcoholic Fatty Liver Development In Vivo. Diabetes 70, 577-588 (2021).

20. Fullerton, M. D. et al. Single phosphorylation sites in Acc1 and Acc2 regulate lipid homeostasis and the insulin-sensitizing effects of metformin. Nat. Med. 19, 1649-1654 (2013).

21. Price, N. L. \& Fernández-Hernando, C. miRNA regulation of white and brown adipose tissue differentiation and function. Biochim. Biophys. Acta 1861, 2104-2110 (2016).

22. Fu, T. et al. MicroRNA 34a inhibits beige and brown fat formation in obesity in part by suppressing adipocyte fibroblast growth factor 21 signaling and SIRT1 function. Mol. Cell. Biol. 34, 4130-4142 (2014).

23. Garton, A. J. et al. Phosphorylation of bovine hormone-sensitive lipase by the AMP-activated protein kinase. A possible antilipolytic mechanism. Eur. J. Biochem. 179, 249-254 (1989).

24. Sullivan, J. E. et al. Inhibition of lipolysis and lipogenesis in isolated rat adipocytes with AICAR, a cellpermeable activator of AMP-activated protein kinase. FEBS Lett. 353, 33-36 (1994).

25. Yin, W., Mu, J. \& Birnbaum, M. J. Role of AMP-activated protein kinase in cyclic AMP-dependent lipolysis in 3T3-L1 adipocytes. J. Biol. Chem. 278, 43074-43080 (2003). 

protein kinase-alpha2 subunit. Diabetes 53, 2242-2249 (2004).

\section{Figures}

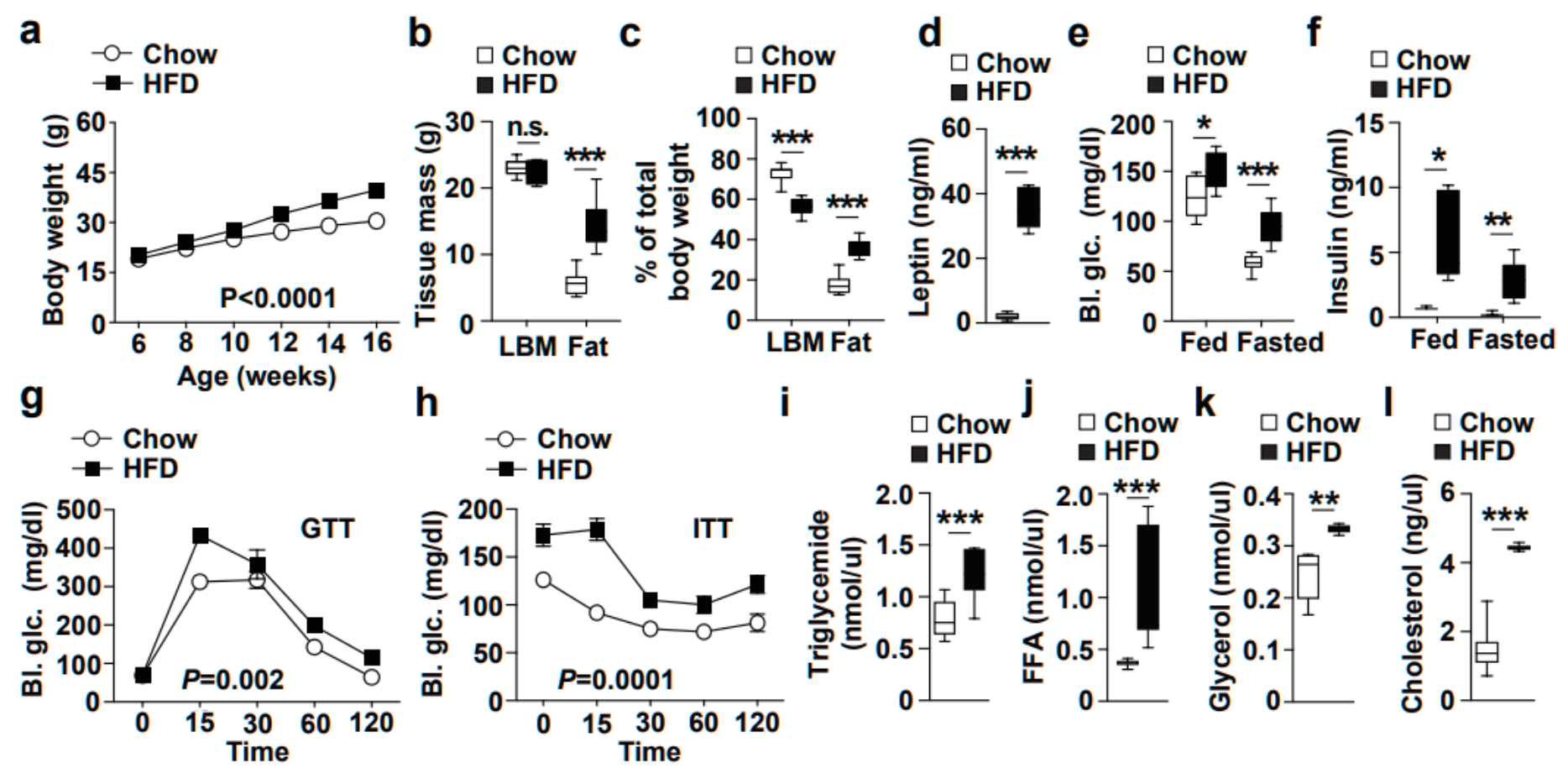

Figure 1

High fat diet results in increased body weight and insulin resistance. a, Body weight curves of HFD fed mice $(n=12)$ and normal chow diet fed controls from 6 to 16 weeks of age. $\mathbf{b}$, Lean body mass and fat mass in 16-week-old HFD and normal chow diet fed mice ( $n=6-11)$. c, Tissue mass to body weight ratio in HFD and control mice ( $n=6-11)$. d, Plasma leptin measurements in 16-week-old HFD and chow diet controls $(n=6-10)$. e, f, Random and fasted plasma glucose and insulin measurement of HFD and Chow mice at 16-week-old age $(n=6,10) . \mathbf{g}, \mathbf{h}$, Glucose measurements during glucose $(\mathbf{g})$ and insulin $(\mathbf{h})$ tolerance test on 12-week-old HFD and normal chow diet fed mice $(n=5-8)$. i-l, Plasma triglyceride, free fatty acid (FFA), glycerol and cholesterol measurement on 16-week-old HFD and normal chow diet fed mice $(\mathrm{n}=6-11)$. Results are presented as mean \pm SEM. ${ }^{\star} P<0.05,{ }^{\star} * P<0.01$ and ${ }^{\star \star \star} P<0.001$. 

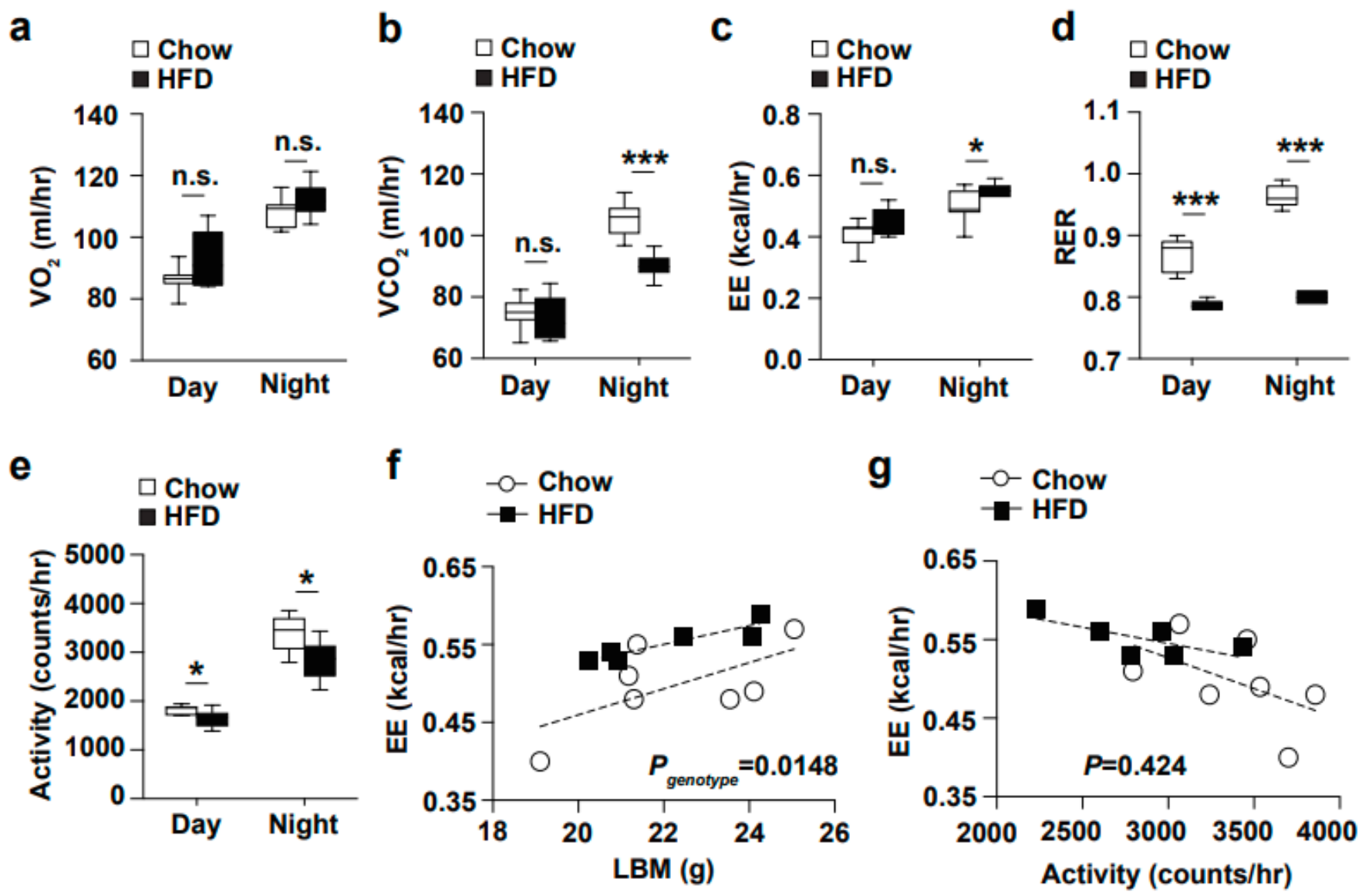

Figure 2

High fat diet alters energy metabolism in mice. a-e, Quantification of $\mathrm{O}_{2}$ production, $\mathrm{CO}_{2}$ consumption, energy expenditure, RER and locomotor activity, respectively in 16-week-old HFD and Chow mice $(n=6-7)$. f, Energy expenditure of individual animals plotted against lean body mass from 16-week-old HFD $(n=6)$ and normal chow diet fed mice $(n=7)$. g, Energy expenditure of individual animals plotted against locomotor activity from 16-week-old HFD $(n=6)$ and normal chow diet fed mice $(n=7)$. Results are presented as mean \pm SEM. ${ }^{\star} P<0.05,{ }^{\star} * P<0.01$ and ${ }^{\star * \star} P<0.001$. 
a

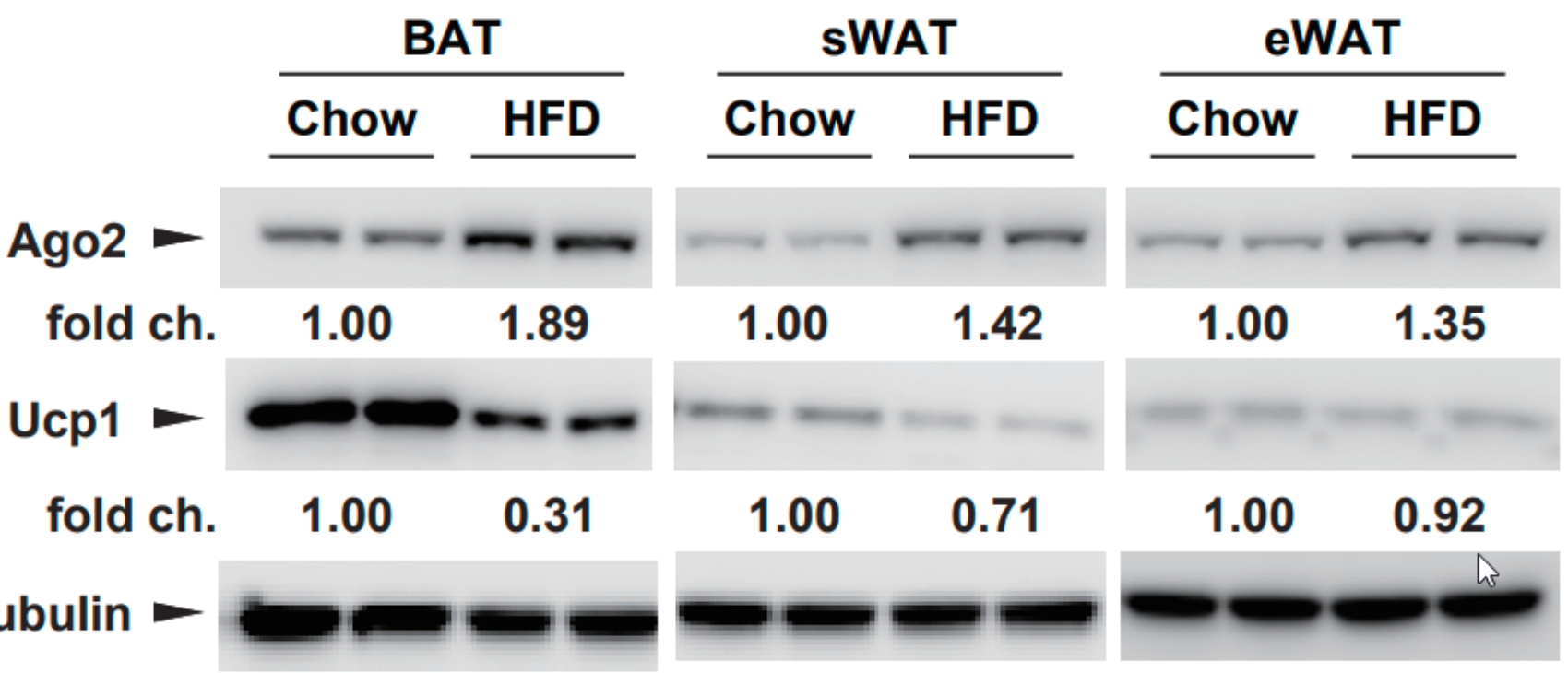

b

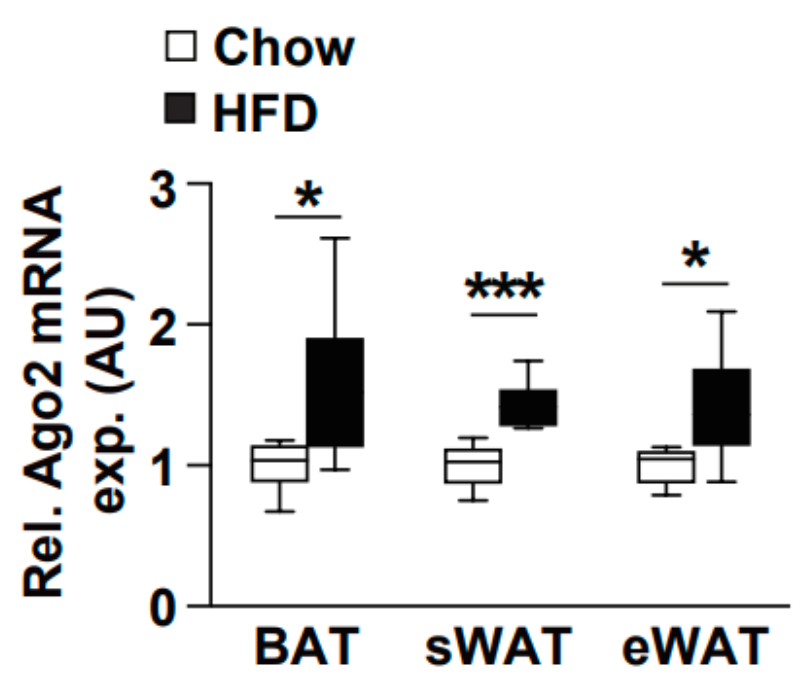

C $\square$ Chow 口 HFD

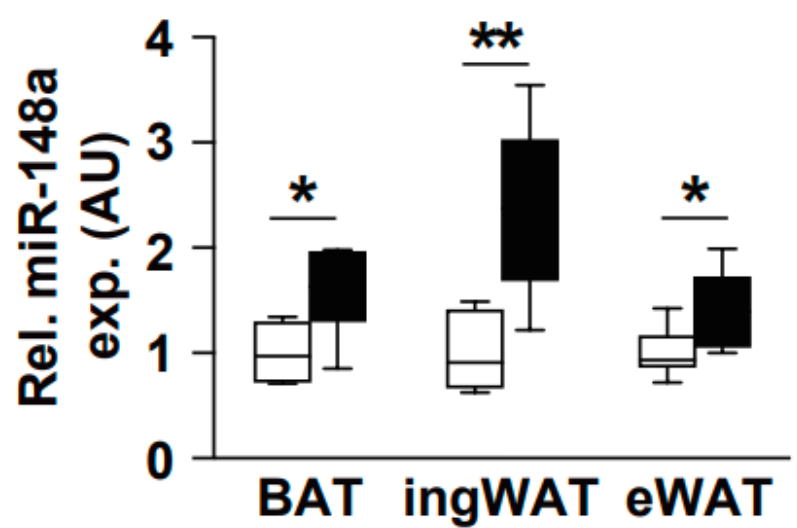

Figure 3

Increased expression of Ago2 and miR-148a in fat tissues of HFD mice. a, Western blot analysis of Ago2 and Ucp1 in total lysate from BAT, sWAT, and eWAT of wild type mice on normal chow diet and littermate controls on HFD feeding. $\mathbf{b}$ and $\mathbf{c}$, qPCR analysis of Ago2 and miR-148a from BAT, sWAT, and eWAT of HFD and Chow mice at 16 weeks old $(n=6)$. Results are presented as mean \pm SEM. ${ }^{\star} P<0.05, \star \star P<0.01$ and $* \star \star P<0.001$. 

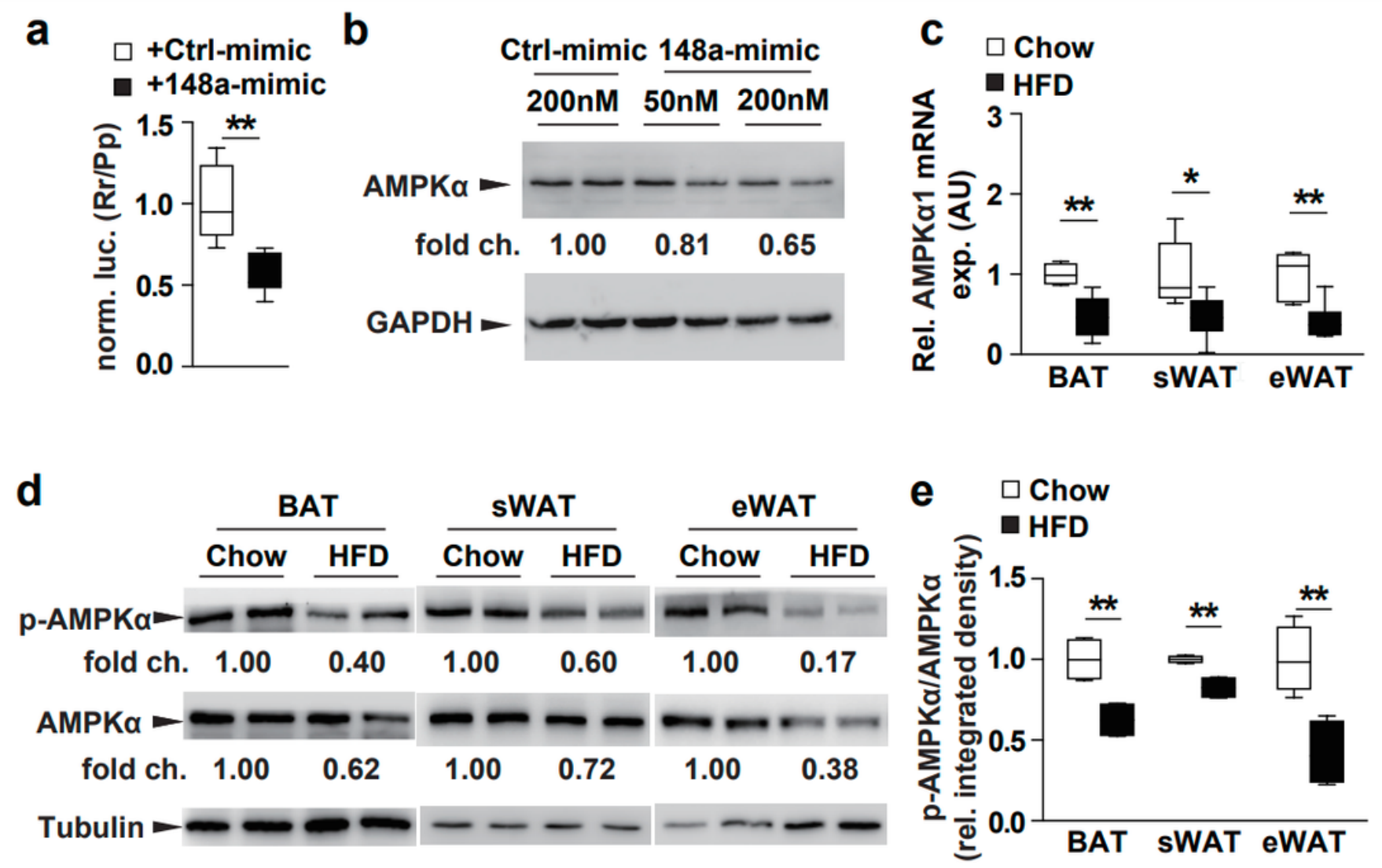

Figure 4

Ago2 regulates miRNA-mediated targeting of AMPKa. a, Luciferase assays in HEK293 cells testing direct targeting of AMPKa genes by miR-148a (148a-mimic). b, Western blot analysis of AMPKa in HEK293 cells after transfection of miR-148a and control mimic. c. qPCR analysis of AMPKa1 from BAT, sWAT, and eWAT of HFD $(n=6)$ and Chow $(n=6)$ mice at 16 weeks old. $d$, Western blot analysis of p-AMPKa and AMPKa from BAT, sWAT, and eWAT of HFD and Chow mice at 16 weeks old. e, Quantification of pAMPKa/AMPKa from BAT, sWAT, and eWAT of HFD and Chow mice at 16 weeks old. Results are presented as mean \pm SEM. ${ }^{*} P<0.05$, and ${ }^{\star *} P<0.01$. 


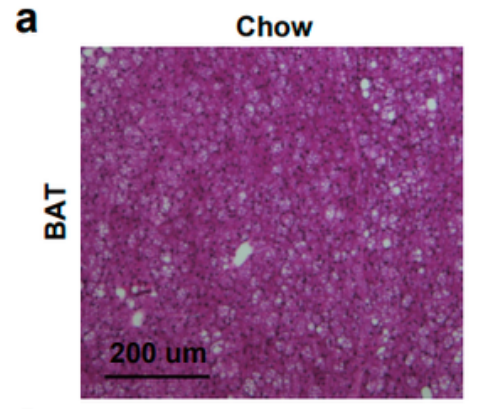

C
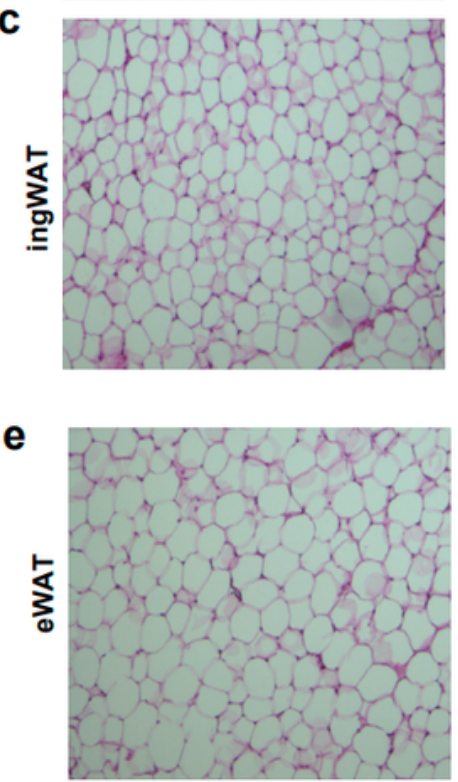

g

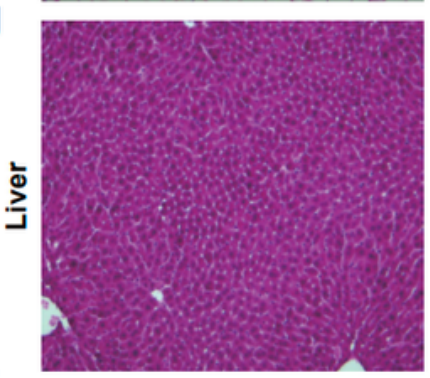

$\square$ Chow $\square$ HFD
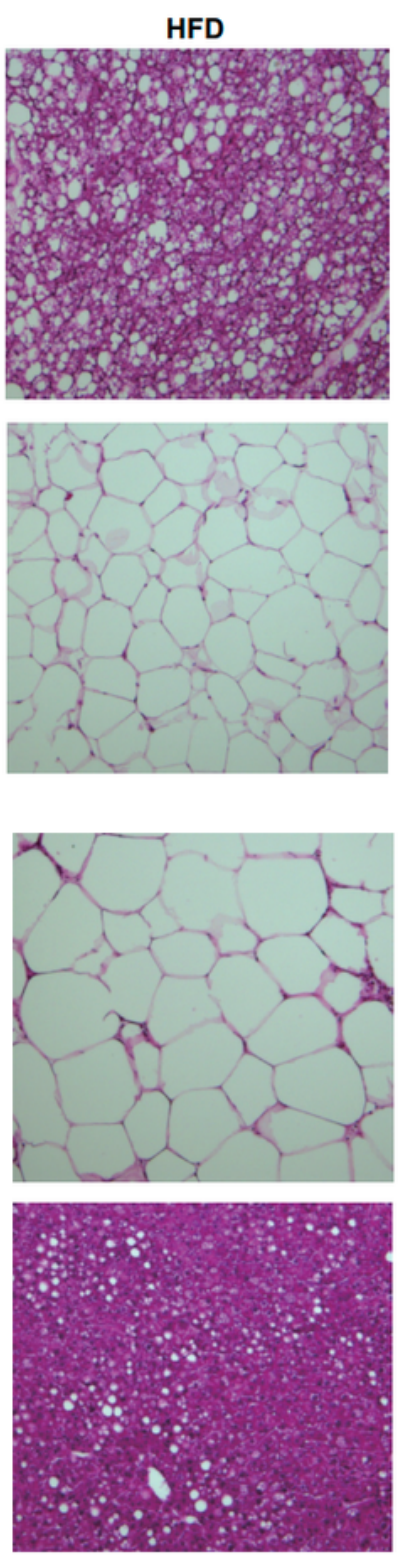

b

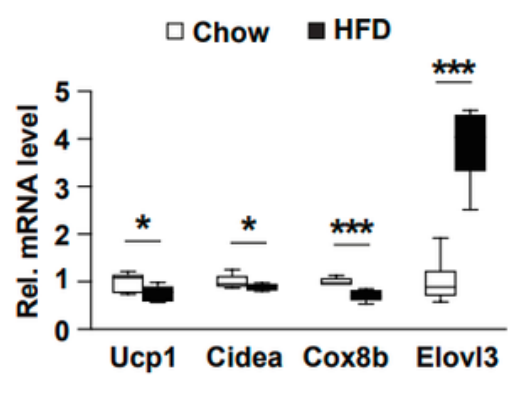

d

$\square$ Chow $\square$ HFD

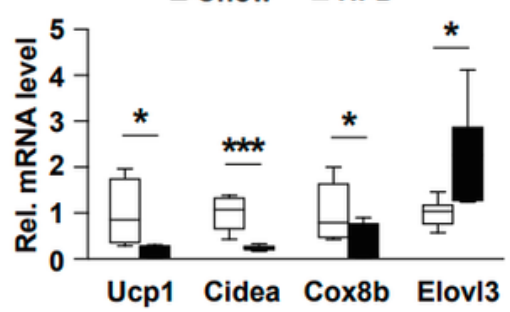

f

h

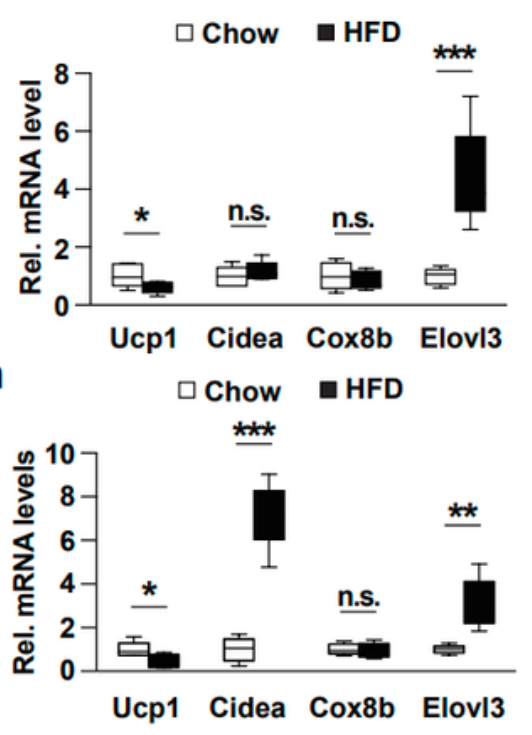

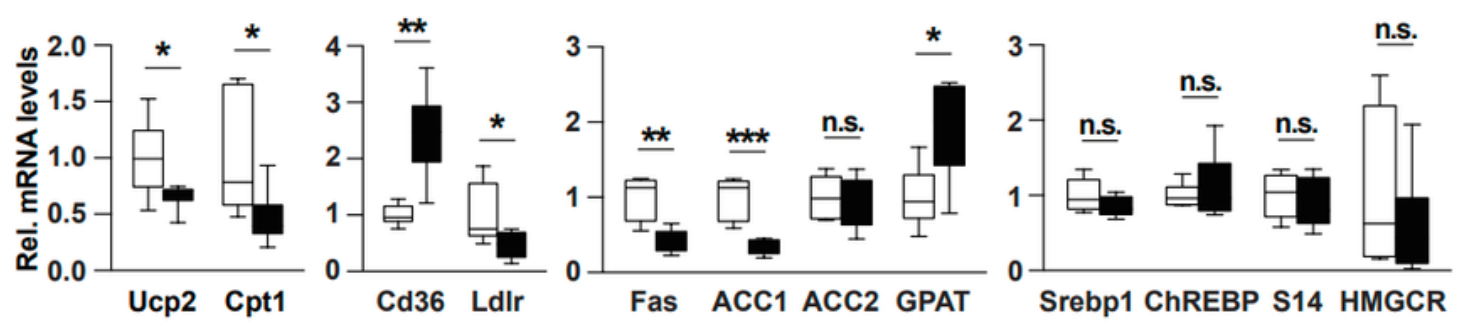

Figure 5

Lipid accumulation in HFD mice. a-h, Haematoxilin and eosin staining of BAT, ingWAT, eWAT and liver and qPCR gene expression analysis from 16-week-old normal chow diet and HFD fed mice $(n=6)$. i, qPCR analysis for gene expression from liver of 16-week-old normal chow diet and HFD fed mice $(n=6)$. Results

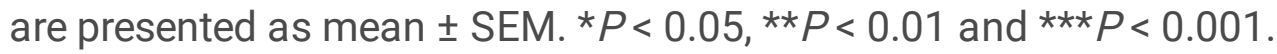




\section{Supplementary Files}

This is a list of supplementary files associated with this preprint. Click to download.

- SupplementaryFigureS1.pdf

- SupplementaryTables1.pdf

- SupplementaryTables2.pdf 